Aim of the study: To discuss the activation of the signal transduction pathway of phosphatidylinositol 3'-kinase/serine-threonine kinase (PI3K/Akt), one of the important targets of drug resistance of trastuzumab, which provides a theoretical basis for the targeted therapy of drug resistance of trastuzumab in breast cancer.

Material and methods: Establish the drug-resistance sub-strain BT-HerR of trastuzumab for the continuous treatment of human breast cancer cell strain BT474, conduct Her-2 phenotype analysis on the drug-resistance cell strain BTHerR with the FISH method, detect the proliferation inhibition in vitro of trastuzumab to BT474 and BT-HerR cells with the MTT method, detect the apoptosis variation after interference of trastuzumab with a flow cytometer and detect p-Akt and apoptosis-related protein expression with Western blot after PI3K/Akt inhibitor LY294002 interferes with the cells.

Results: The gene expression of drug-resistance cell strain BT-HerR Her-2 is strongly positive; 72 hours after interference of trastuzumab, the proliferation in vitro of the BT474 and BT-HerR cells is inhibited, which is strengthened with the increase of concentration, showing a significant difference $(p<0.01)$; after treatment of trastuzumab, comparison of the cell apoptosis rate of BT474 and BT-HerR shows a significant difference $(p<0.01)$; trastuzumab can only inhibit the Akt protein phosphorylation of BT474, while LY294002 can inhibit the BT-HerR and BT474 Akt protein phosphorylation simultaneously.

Conclusions: Akt protein phosphorylation of trastuzumab drug-resistance cells is activated; LY294002, a PI3K/Akt inhibitor, can obviously inhibit Akt protein phosphorylation of trastuzumab drug-resistance cells and there is a clear association between the PI3K/Akt signal transduction pathway and trastuzumab resistance.

Key words: trastuzumab, LY294002, PI3K/Akt, breast cancer cell, HER2/neu.

Contemp Oncol (Pozn) 2013; 17 (4): 363-369 DOI: $10.5114 /$ wo.2013.35292

\section{Research on drug resistance mechanism of trastuzumab caused by activation of the PI3K/Akt signaling pathway}

\author{
Xi Chen, Han Wang, Xue-nong Ou-yang, Fang-wei Xie, Jing-jing Wu
}

Department of Medical Oncology, Fuzhou General Hospital of Nanjing Military Command, Fuzhou, China

Breast cancer is one of the most common female malignant tumors, with morbidity increasing year by year and age of onset tending to be younger, so its harm cannot be neglected. The HER2/neu gene (also called c-erbB2) coding transmembrane receptor tyrosine protein kinase has an overexpression rate of up to $30 \%$ in breast cancer and is closely related to adverse prognosis and low sensitivity to radiotherapy and chemotherapy [1, 2]; therefore targeted therapy against HER-2/neu has become a research hotspot in recent years. Trastuzumab (trade name: Herceptin) is a kind of humanized monoclonal antibody resisting the HER-2/neu gene. In 1998, upon the approval of the Food and Drug Administration (FDA), it was used for the first-line treatment of Her-2 positive advanced breast cancer. Trastuzumab has a good treatment effect on Her-2 positive breast cancer and obviously extends the disease-free survival of patients; however, nearly $50 \%$ of Her-2 positive patients are not sensitive to trastuzumab treatment [3]. After 1 year's trastuzumab treatment, most Her-2 positive patients will generate drug resistance to trastuzumab. The drugresistance mechanism of trastuzumab is relatively complex [4]. The activation of the phosphatidylinositol 3'-kinase/serine-threonine kinase (PI3K/Akt) signal transduction pathway is deemed as one of the important mechanisms of trastuzumab drug resistance [5,6]. With the important gene p-Akt expression that drug-resistance mechanism might occur to trastuzumab in the PI3K signaling pathway as the entry point and through the inhibition of PI3K/Akt activity of trastuzumab drug-resistance breast cancer cells by its inhibitor LY294002. This research aims to discuss the drug-resistance mechanism of trastuzumab, thus providing a theoretical basis for further improving the treatment effect of breast cancer trastuzumab targeted treatment.

\section{Material and methods}

\section{Main experiment reagents}

Human breast cancer cell strain BT474 was purchased from Chinese Academy of Science Shanghai Cell Bank, trastuzumab from Roche, RPMI 1640 culture medium and fetal calf serum from American HyClone, EDTA-trypsin from American Gibco, penicillin streptomycin solution from Shanghai Beyotime Biotechnology Co., Ltd., $25 \mathrm{~cm}^{2}$ cell culture bottle, 96-hole and 6-hole culture plate from American Corning, dimethyl sulfoxide (DMSO) and MTT from American Sigma, AnnexinV - FITC/PI apoptosis kit from American BioVision, RIPA lysate, PMSF, prestained protein marker, horseradish peroxidase marker goat anti-rabbit IgG, ECL chemiluminescence kit from Shanghai Beyotime Biotechnology Co., Ltd., and anti-rabbit human phosphorylation protein kinase B (p-AKT) monoclonal antibody, PI3K inhibitor LY294002 and anti-rabbit human $\beta$-actin monoclonal antibody all from American Cell Signaling. In situ hybridization reagents (including HER-2/CSP17DNA probe kit, $2 \times$ SSC buffer 
solution, protease K, 10\% NP-40 and DAPI after stain) were purchased from Beijing GP Medical Technologies, Ltd.

\section{Main experiment equipment}

Scientific Model 1205A-type clean bench, Scientific Model 3111-type $\mathrm{CO}_{2}$ incubator (American FORMA), Model 680type micro plate reader (Raito Life and Analytical Sciences Co., Ltd.), CKX31-type inverted phase contrast microscope (Japanese OLYMPUS), Epics XL-type flow cytometer (American Beckman Coulter), Power PAC 300-type vertical electrophoresis apparatus, Power PAC200 electro orator and Fluor-S gel imaging analysis system (American Bio-Rad).

\section{Cell culture}

Apply conventional anchorage-dependent cell culture method to culture the cells in RPMI 1640 culture solution containing $10 \%$ fetal calf serum and then place them in an incubator containing $5 \% \mathrm{CO}_{2}$ at $37^{\circ} \mathrm{C}$. Use $0.25 \%$ trypsin for digestion and passage.

\section{Method of induction to create drug-resistance cell} strain [7]

Through 6 months' continuous treatment of BT474, BTHerR, a drug-resistance sub-strain resisting trastuzumab, is created. From $1 \mu \mathrm{g} / \mathrm{ml}$, the sub-strain is cultured continuously to its stable growth in low-concentration trastuzumab and its morphology is basically consistent with that of the parental generation. Then the concentration of trastuzumab in culture solution is increased gradually to $10 \mu \mathrm{g} / \mathrm{ml}$. Maintain the concentration during culture, and change the culture solution once every 4 days. Conduct passage when the density reaches $70-80 \%$. Identify the drug resistance of the sub-strain by using the MTT method.

\section{Her-2 phenotype analysis of drug-resistance strain in FISH method [8]}

Pretreatment before FISH: dissociate cells to prepare $1 \times$ $\times 105 / \mathrm{ml}$ cell suspension, take $5-10 \mathrm{ml}$ for centrifugation at 1000 RPM for 10 min and abandon the supernatant. Then, add $5 \mathrm{ml} 0.56 \% \mathrm{KCL}$ solution at $37^{\circ} \mathrm{C}$, blow and beat the resuspended cells again and incubate the cells in a water bath at $37^{\circ} \mathrm{C}$ for $20 \mathrm{~min}$. Add $2 \mathrm{ml}$ of stationary liquid to the test tube, centrifuge it at 1500 RPM for 10 min, suck and abandon the supernatant to $1 \mathrm{ml}$, add $5 \mathrm{ml}$ of stationary liquid cell re-suspension and place it still at room temperature for 10 min; after that, centrifuge it at 1500 RPM for 10 min again, suck and abandon the supernatant to $1 \mathrm{ml}$, then add $5 \mathrm{ml}$ of stationary liquid cell re-suspension and place it still at room temperature for $10 \mathrm{~min}$. Centrifuge at 1000 RPM for $10 \mathrm{~min}$, suck and abandon the supernatant to $1 \mathrm{ml}$, drop the cells on a slide after blow, beat and re-suspension. Age the slide at $56^{\circ} \mathrm{C}$ for $30 \mathrm{~min}$.

Hybridization: When the slide has been air dried, drip $10 \mu$ l from the probe to the area for hybridization, then cover it with a cover glass and seal the edge with rubber glue. Place it into an automatic hybridization oven to treat at a denaturalization temperature of $75^{\circ} \mathrm{C}$ for 5 min and keep at $42^{\circ} \mathrm{C}$ overnight. Washing after hybridization: remove the cover glass and wash the slide in $2 \times$ SSC solution twice, each time for $10 \mathrm{~min}$, and then wash in $2 \times \mathrm{SSC} / 0.1 \% \mathrm{NP}-40$ solution for $5 \mathrm{~min}$. Then, soak in $70 \%$ ethanol solution for 3 min and place the slide in a dark place for air drying. Add 10-20 $\mu$ l of 4',6-diamidino-2-phenylindole (DAPI) to all sections, cover them with a cover glass before placing them in a dark place for $10 \mathrm{~min}$, and then observe through a special single-channel filter by a fluorescence microscope.

Result assessment: count 30 cells and perform statistics of the ratio value (Ratio value $=$ total amount of red signal in 30 cell nuclei/total amount of green signal in 30 cell nuclei). A result with a ratio $<1.8$ is negative, which indicates no gene amplification of HER-2 in the sample; a result with a ratio $>2.2$ is positive, which indicates a gene amplification of HER 2 in the sample; where the ratio is between 1.8 and 2.2, increase the number of counted cells to 100 or repeat the FISH test to determine the final result; in case of aggregation of abnormal cells or appearance of multiple cells with a red signal of each cell nucleus $>10-15$, it should be treated as a positive result. If the green signal in each nucleus is $>2.25$, it should be considered as a polysomic chromosome.

\section{Cell proliferation inhibition detection in MTT method [7]}

Collect cells at exponential phase, adjust the cell density to $3 \times 10^{4} / \mathrm{ml}$ and inoculate $100 \mu$ l to each hole on a 96 hole culture plate. After 24 hours, add drugs of different concentrations, while setting the zero-setting hole (do not inoculate the cells but add corresponding cell culture fluid) and negative control groups with 6 complex holes set for each group. Incubate in atmosphere of $5 \% \mathrm{CO}_{2}$ at $37^{\circ} \mathrm{C}$ for 5 days with the solution changed once during this period. After a 5-day intervention of trastuzumab, add $10 \mu$ l of MTT solution to each hole of the 96 -hole culture plate $(5 \mathrm{mg} / \mathrm{ml})$ and continue to culture for 4 hours before stopping to carefully absorb the culture fluid in the holes. Add $100 \mu$ l of DMSO to each hole and put the plate on a shaker to vibrate for $10 \mathrm{~min}$ so as to fully dissolve the crystals. Measure the absorbance of each hole at OD490 nm of the ELISA Reader. Meanwhile, set zero-setting holes (added with medium, MTT and DMSO), and control holes (added with cells, drug dissolution medium with the same concentration, culture fluid, MTT and DMSO). The formula adopted for the calculation of the inhibition ratio of cell proliferation is:

inhibition ratio $=100 \% \times[(O D$ value of control hole $-O D$ value of zerosetting hole) - (OD value of dosing hole - OD value of zero-setting hole)]/ (OD value of control hole - OD value of zero-setting hole).

\section{Repeat the experiment 3 times.}

\section{Detection of cell apoptosis by flow cytometer}

After the cells are treated, collect all anchorage-dependent cells and floating cells to the flow cytometer for centrifugation at 1000 RPM for 5 min. Wash the cells with PBS twice and record the amount. Use $1 \times$ Binding Buffer to resuspend the cells, adjust the cell concentration to $1 \times 10^{6} / \mathrm{ml}$, drip $100 \mu$ l of cell suspension to a $5 \mathrm{ml}$ flow tube, and then add Annexin $\vee 5 \mu$ land PI $10 \mu$ l to gently blend it and keep in a dark place for $10 \mathrm{~min}$ at room temperature. Immediately 
detect and analyze the result to calculate the percentage of the viable apoptotic cell.

Detection of p-Akt of cells and apoptosis related protein expression by Western blot [9]

Collect cells and add cell lysate before placing on ice for dissociation for $30 \mathrm{~min}$, and then centrifuge it at 12000 $\mathrm{RPM} / \mathrm{min}$ for $20 \mathrm{~min}$ at $4^{\circ} \mathrm{C}$. Retain the supernatant, take protein with the equal amount to conduct SDS-PAGE denatured gel electrophoresis, and then transfer protein to PVDF membrane. Seal the 5\% skim milk at room temperature for $2 \mathrm{~h}$ and combine with the first antibody overnight. After the membrane is washed, combine it with the secondary antibody for $2 \mathrm{~h}$. Then detect in chemiluminescence method to expose the film, wash down and dry up. Analyze the grey level on the stripe in the picture by Quantity One software through scanning of the gel imaging system. Repeat the procedure 3 times.

\section{Statistical analysis}

The measured data are presented as mean \pm standard deviation $(\bar{x} \pm s)$. We used the t-test to compare the means of two samples and analyzed the dose-effect relationship using Spearman rank correlation; the variance analysis was conducted using the statistical software SPSS 13.0. All the results indicating $p<0.05$ show statistical significance.

\section{Results}

Establishment of cell line of BT-HerR with drug resistance to trastuzumab

FISH test result of cell line of BT-HerR with drug resistance to trastuzumab See in Fig. 1.

\section{Inhibition of in vitro proliferation of BT474 and BT-HerR cells by trastuzumab}

It is shown in the result of the MTT method that after trastuzumab with a concentration between 0.1 and $100 \mu \mathrm{g} / \mathrm{ml}$ interferes with cells for 72 hours, the in vitro proliferation of the cells is inhibited with the effect on the proliferation increasing with the rising of the concentration. Through calculation, the IC50 of trastuzumab on BT474 cells and drug-resistance cell strain BT-HerR was $1.336 \mathrm{mg} / \mathrm{l}$ and $33.994 \mathrm{mg} / \mathrm{l}$, respectively; there was no significant difference $(p<0.01)$. The pairwise comparison difference between experimental groups with different concentrations has statistical significance $(p<0.01$; Table 1$)$
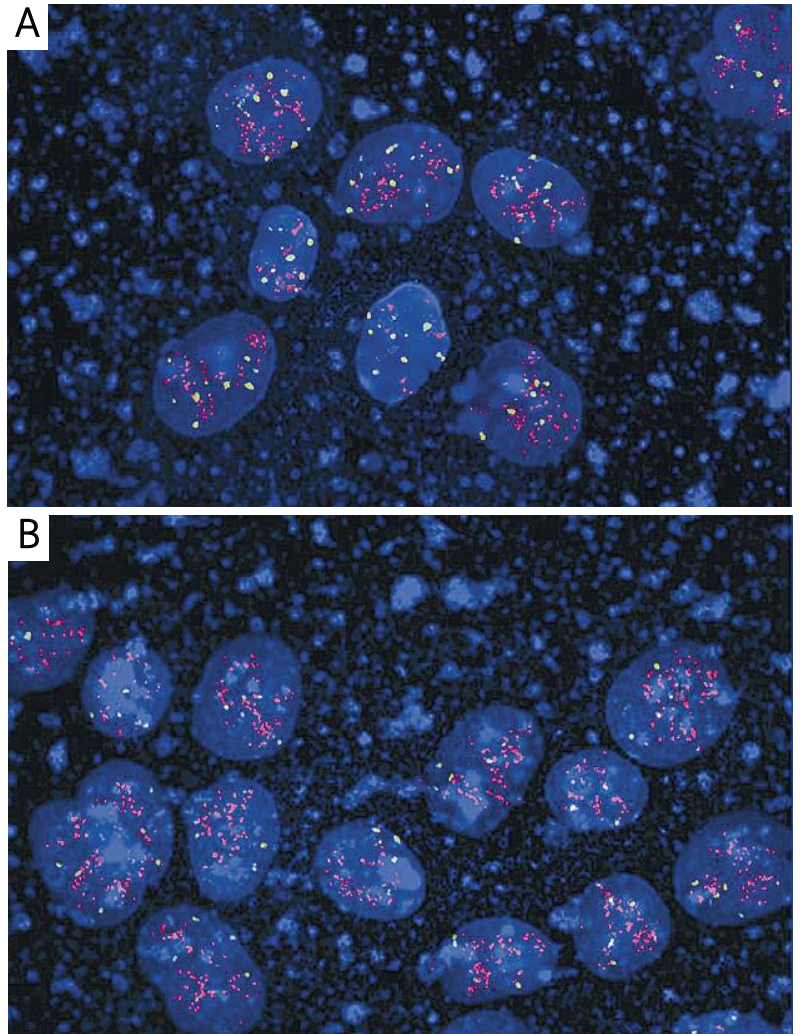

Fig. 1. Picture A is a BT474 cell, Picture B is a BT-HerR cell. Her-2 gene amplification detection kit contains 2 DNA probes: GLP HER-2 and CSP 17. The fluorescent signal of the Her-2 DNA probe is jacinth and as a contrast to gene amplification, the fluorescent signal of CSP 17 is green. The ratio of the two signals is used as the basis to judge positive or negative. In this respective, it can be seen that in picture B Her-2 genes amplify in a form of cluster, which indicates strongly positive

\section{Cell apoptosis of BT474 and BT-HerR under the interference of trastuzumab}

As shown in the test result of flow cytometry, after BT474 cells are interfered with by $10 \mu \mathrm{g} / \mathrm{ml}$ trastuzumab, apoptosis occurs (Fig. 2); the difference between apoptosis rate of BT474 and BT-HerR has statistical significance $(p<0.01$; Table 2).

\section{Influence on BT474 and BT-HerR by PI3K/Akt} inhibitor LY294002 and trastuzumab

In order to observe the influence of PI3K/Akt pathway inhibition on drug resistance of trastuzumab in breast cancer cells, treat the cells with $10 \mu \mathrm{mol} / \mathrm{I} \mathrm{PI3K} / \mathrm{Akt}$ inhibitor LY294002 for $6 \mathrm{~h}$ in advance, then add $10 \mu \mathrm{g} / \mathrm{ml}$ trastuzumab to treat the

Table 1. Inhibition ratio on cell proliferation of BT474 and BT-HerR under the interference of trastuzumab with different concentrations for 5 days $(\%, \bar{x} \pm \mathrm{s})$

\begin{tabular}{lcccc} 
Cell line & \multicolumn{4}{c}{ Concentration of Trastuzumab $(\mu g / \mathrm{ml})$} \\
\cline { 2 - 5 } & 0.1 & 1 & 10 & 100 \\
BT474 & $10.17 \pm 1.13$ & $35.95 \pm 2.56$ & $71.97 \pm 3.57$ & $79.71 \pm 1.49$ \\
BT-HerR & $3.34 \pm 0.94$ & $9.59 \pm 2.61$ & $21.34 \pm 1.60$ & $32.73 \pm 2.44$ \\
\hline
\end{tabular}

Pairwise comparison between experimental groups with different concentrations $(p<0.01)$ 
A

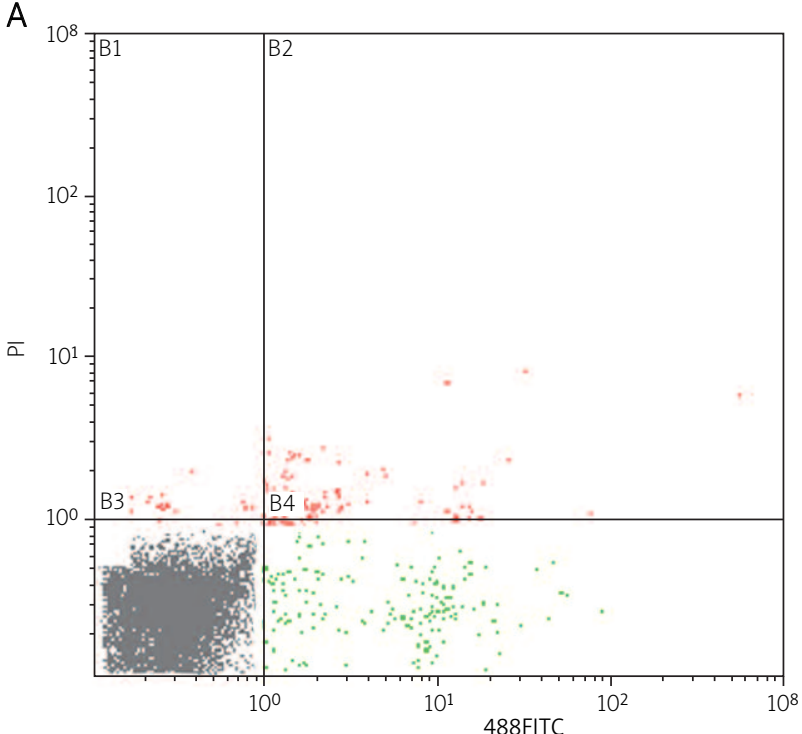

C

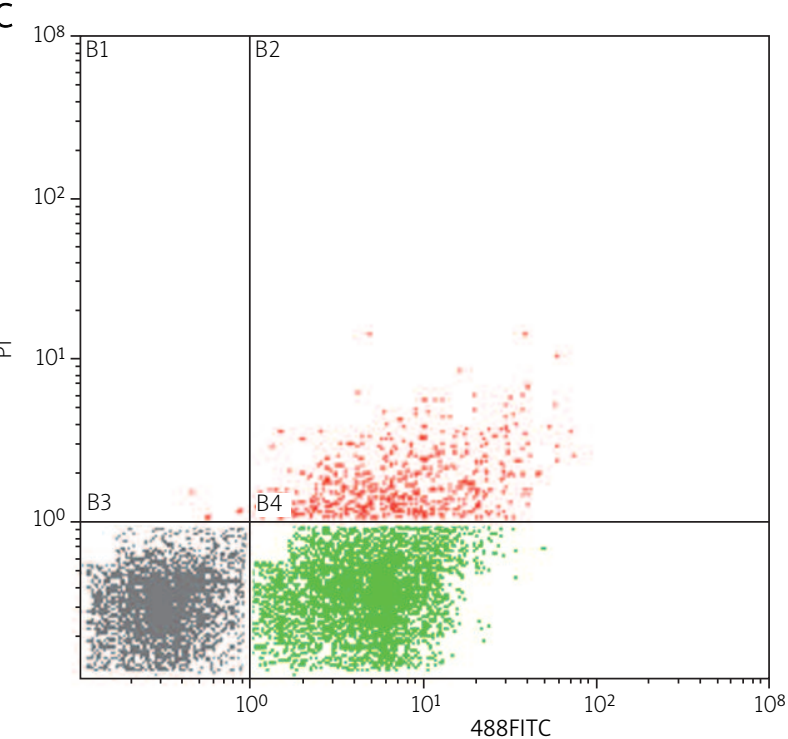

B

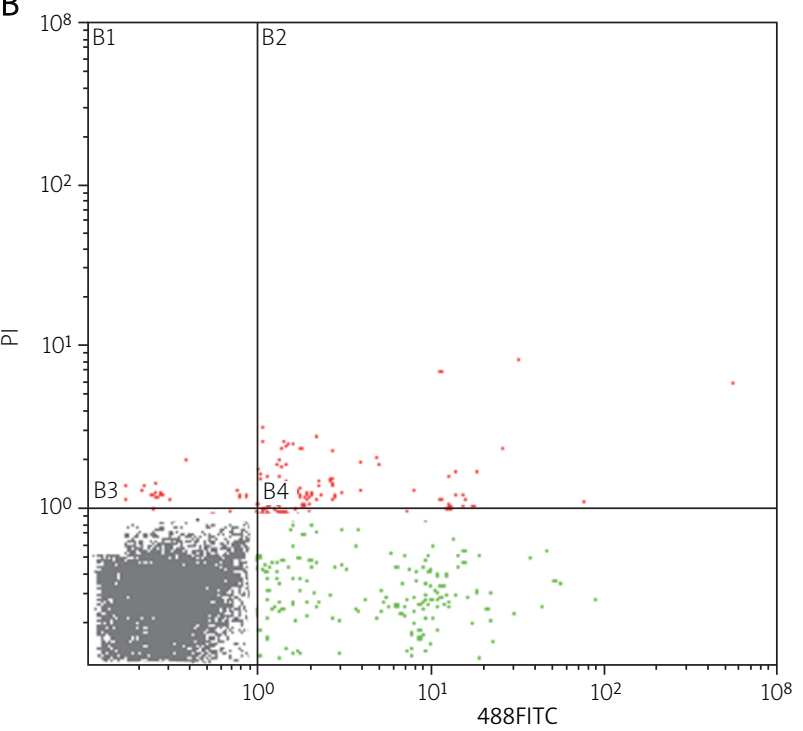

D

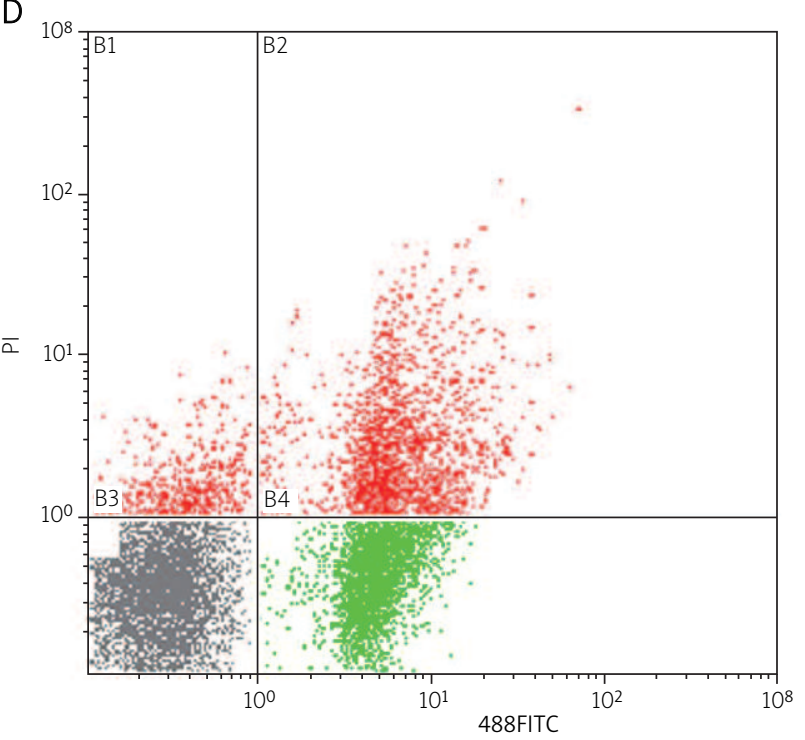

Fig. 2. A) Untreated cells of BT474; B) Untreated cells of BT-HerR; C) BT474 cells treated with trastuzumab; D) BT-HerR cells treated with trastuzumab

Table 2. Apoptosis rate of BT474 and BT-HerR under the interference of $10 \mu \mathrm{g} / \mathrm{ml}$ trastuzumab $(\%, \bar{x} \pm \mathrm{s})$

\begin{tabular}{lcccc} 
Group & Cell line & $\begin{array}{c}\text { Apoptosis rate } \\
\text { in early stage (B4) }\end{array}$ & $\begin{array}{c}\text { Apoptosis rate and } \\
\text { mortality rate in middle- } \\
\text { advanced stage (B2) }\end{array}$ & Survival rate (B3) \\
\hline Blank control & BT474 & $3.38 \pm 0.08$ & $0.58 \pm 0.02$ & $95.34 \pm 1.07$ \\
\hline Treated with trastuzumab & BT-HerR & $3.34 \pm 0.17$ & $1.68 \pm 0.07$ & $94.98 \pm 1.23$ \\
& BT474 & $24.58 \pm 0.55^{*}$ & $3.60 \pm 0.17$ & $71.76 \pm 2.17$ \\
& BT-HerR & $6.97 \pm 0.24^{*}$ & $1.84 \pm 0.14$ & $89.07 \pm 1.52$
\end{tabular}

Comparison of apoptosis rate of BT474 and BT-HerR after being treated with trastuzumab, ${ }^{*} p<0.01$

cells. Observe whether the apoptosis of drug-resistance cells of BT-HerR changes or not.

\section{Influence on the apoptosis rate of BT474} and BT-HerR by LY294002 and trastuzumab

As shown in the test result of flow cytometry, there is obvious apoptosis in BT474 and BT-HerR after being treated by LY294002 in advance and added with trastuzumab later. The difference between the apoptosis rate of BT474 and drugresistance BT-HerR has no statistical significance.

\section{Influence on p-Akt protein expression in BT474} and BT-HerR cells by LY294002 and trastuzumab

In order to observe the effect of $\mathrm{p}$-Akt in drug resistance of trastuzumab, respectively treat BT474 and BT-HerR cells with LY294002 and trastuzumab, and collect cell samples to con- 

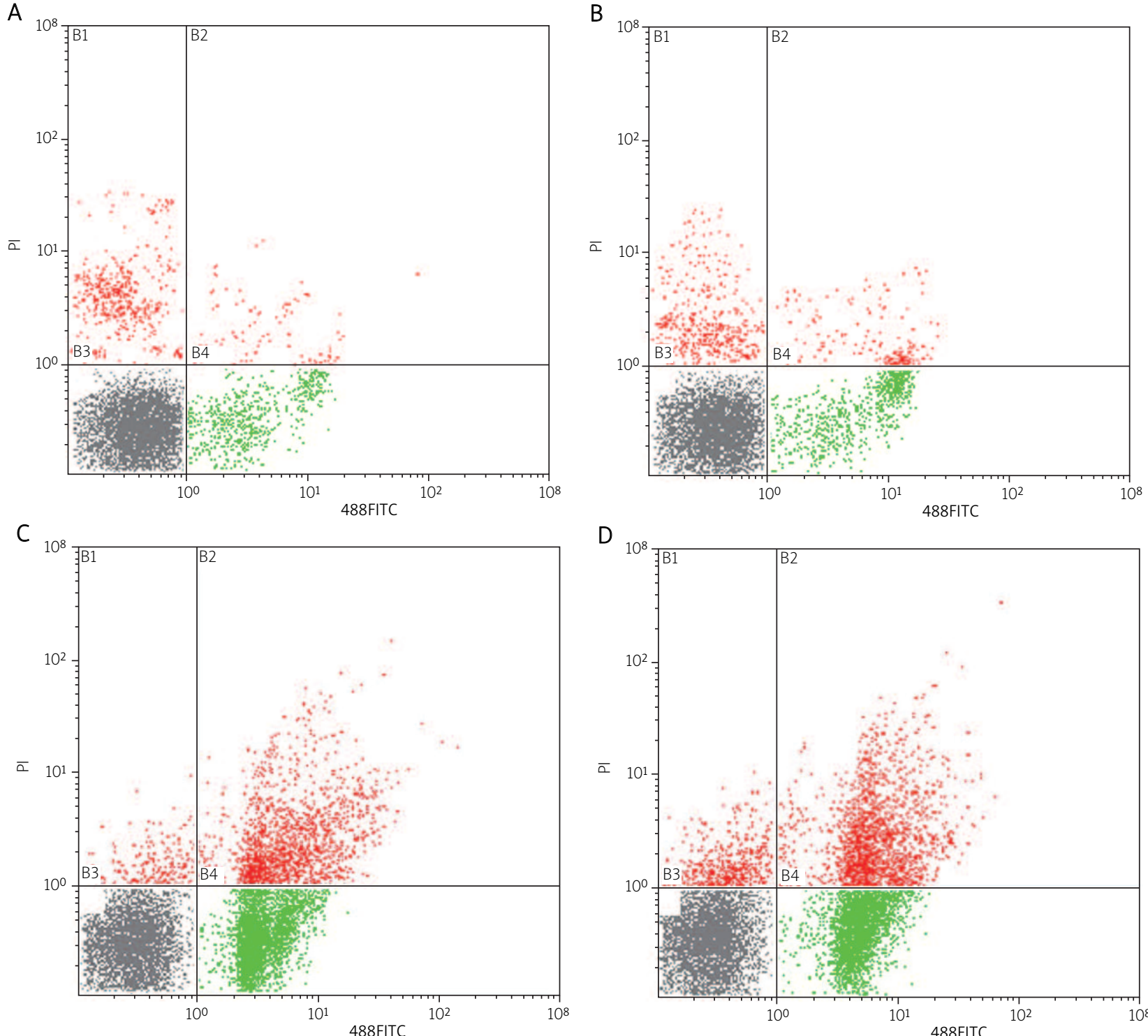

Fig. 3. A) BT474 cells treated with LY294002; B) BT-HerR cells treated with LY294002; C) BT474 cells treated with LY294002 with trastuzumab; D) BT-HerR cells treated with LY294002 with trastuzumab

Table 3. Apoptosis rate of BT474 and BT-HerR cells with or without joint interference of LY294002 and trastuzumab (\%, $\bar{x} \pm s)$

\begin{tabular}{lcccc} 
Group & Cell line & $\begin{array}{c}\text { Apoptosis rate } \\
\text { in early stage (B4) }\end{array}$ & $\begin{array}{c}\text { Apoptosis rate and } \\
\text { mortality rate in middle- } \\
\text { advanced stage (B2) }\end{array}$ & Survival rate (B3) \\
\hline LY294002 group & BT474 & $13.71 \pm 0.67$ & $3.98 \pm 0.03$ & $74.38 \pm 2.31$ \\
& BT-HerR & $10.29 \pm 1.10$ & $1.51 \pm 0.06$ & $79.33 \pm 4.02$ \\
LY294002 with trastuzumab & BT474 & $36.42 \pm 2.32$ & $17.44 \pm 1.32$ & $44.01 \pm 3.60$ \\
& BT-HerR & $32.24 \pm 1.74$ & $19.61 \pm 1.22$ & $42.65 \pm 2.68$
\end{tabular}

duct Western blot test so as to observe the changes in their P-Akt. It can be seen that $10 \mu \mathrm{g} / \mathrm{ml}$ trastuzumab inhibits the Akt protein phosphorylation of BT474, but it cannot inhibit the Akt protein phosphorylation of BT-HerR. However, $10 \mu \mathrm{mol} / \mathrm{l}$ LY294002 can inhibit the Akt protein phosphorylation of both.

\section{Discussion}

The drug-resistance mechanism of trastuzumab has presently become a hot issue in research, wherein research on the drug-resistance target spot is considered as the leading priority. Akt is an important downstream target kinase in the PI3K signal transduction pathway. The PI3K/Akt signaling pathway induces the cell to proliferate and differentiate so as to prevent cell apoptosis. This signaling pathway, as an important transduction pathway for cell growth and proliferation, plays an important role in keeping cell malignant biological characteristics [10-12]; therefore, the inhibition of this signaling pathway has become a hot issue for tumor pre- 


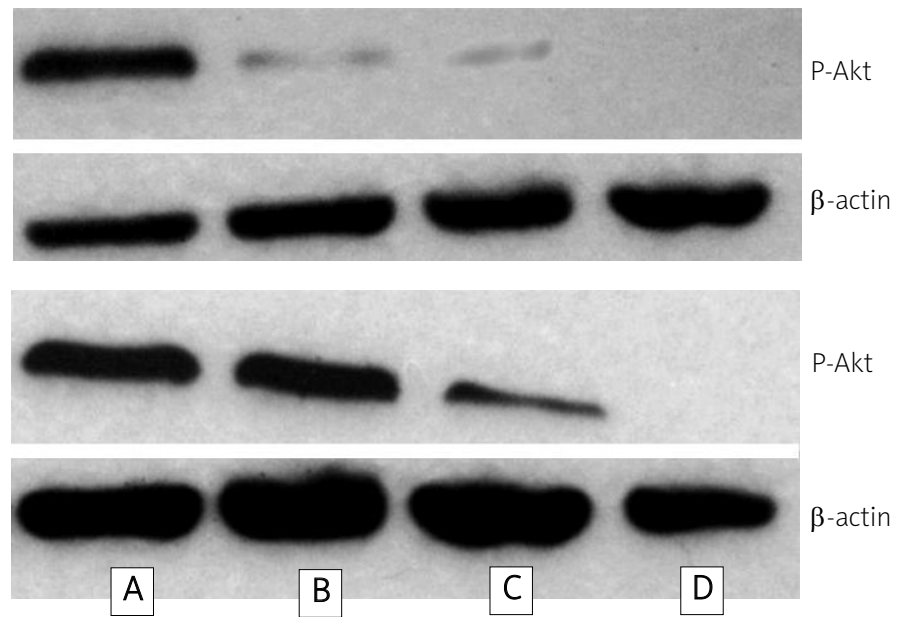

Fig. 4. Influence on expression of BT474 and BT-HerR by PI3K/Akt inhibitor LY294002 and trastuzumab. Above are cells of BT474 and below are cells of BT-HerR. The conditions of sample injection are as follows: A) Untreated blank control group; B) Treat with $10 \mu \mathrm{g} / \mathrm{ml}$ trastuzumab for 24 hours; C) Treat the cells for $6 \mathrm{~h}$ with $10 \mu \mathrm{mol} / \mathrm{l} \mathrm{LY294002;} \mathrm{D)} \mathrm{Add} 10 \mu \mathrm{g} / \mathrm{ml}$ trastuzumab to jointly treat for $24 \mathrm{~h}$ after treating with $10 \mu \mathrm{mol} / \mathrm{l} \mathrm{LY} 294002$ for $6 \mathrm{~h}$

vention and tumor targeted therapy. One of the important functional mechanisms of trastuzumab is to restrain the signal transduction of HER2-PI3K-Akt. The combination of trastuzumab and HER2 can restrain HER3 comprised tyrosine phosphorylation and disturb the formation of HER3 and HER2 heterodimer and HER3 and the PI3Kp85 regulator subunit, so as to restrain the activation of PI3K and reduce Akt phosphorylation $[13,14]$. By restraining the PI3K-Akt pathway, trastuzumab can restrain the formation of breast cancer cell clone through HER2 gene amplification and down-regulate cyclin, thereby inducing cancer cell apoptosis. However, at the same time, many cell factors can activate Akt, such as EGF, IGF-1, heregulin and FGF, insulin, interleukin 3 (IL-3), IL-6 as well as VEGF, etc. Clark [15] et al. found that Akt of some breast cancer cell strains has high intrinsic activity and the HER-2 positive tumor expresses high-level p-AKT. Furthermore, the continuously activated Akt will lead to a deficiency of cell growth inhibition mediated by trastuzumab, and restrain cell cycle arrest or cell apoptosis mediated by trastuzumab. Therefore, continuous activation of Akt may exist in a trastuzumab drug-resistance cell strain, which may be one of the factors that make trastuzumab resist the drug. Lots of inhibitors, targeted to PI3K-AKt, make PI3K inactivate, restrain the proliferation of trastuzumab drug-resistance cells and accelerate their apoptosis and reverse the resistance of trastuzumab, which indicates that PI3K targeted therapy may overcome trastuzumab resistance [16]. LY294002, transformed from quercetin, is a typical PI3K inhibitor. The inhibitor of targeted PI3K's catalytic subunit p110 can effectively restrain the growth of tumor cells in vivo and in vitro, block the PI3K/Akt signaling pathway upstream in a non-specific way, and thereby induce tumor cell apoptosis $[17,18]$. The present research on the drug-resistance mechanism of trastuzumab is still in exploration. The runaway PI3K/Akt signaling pathway and continuous activation state of $p$-Akt are just two important target spots for drug resistance of trastuzumab, which need to be further investigated in future experiments.
After the continuous treatment for human breast cancer cell BT474, the drug-resistance sub-strain BT-HerR that resists the trastuzumab is created. After the treatment with trastuzumab, comparison of the cell apoptosis rate of BT474 and BT-HerR shows a significant difference. Also the apoptosis rate of the two cells shows a significant difference. After trastuzumab drug-resistance BT-HerR cells are activated through Akt protein phosphorylation, the activity of trastuzumab drug-resistance breast cancer cell PI3K/AKt is restrained through the PI3K/Akt inhibitor which is LYA294002, which proves that trastuzumab can restrain the Akt protein phosphorylation of BT474, but cannot restrain that of the BT-HerR cell. However, LY294002 can restrain the Akt protein phosphorylation of both cells, which further proves that LY294002 restrains the activity of PI3K/Akt, and can also reverse the resistance effect of trastuzumab drug-resistance breast cancer cells to trastuzumab through LYA294002. The specific dependence of the existence of the PI3K/Akt path and trastuzumab drug resistance further explicates the position of the target spot.

To sum up, this experiment takes the important gene expression $\mathrm{p}$-Akt on the PI3K signaling pathway where a drugresistance mechanism may occur to trastuzumab as an entry point, adopts the PI3K/Akt inhibitor LY294002 to inhibit the activity of the trastuzumab drug-resistance breast cancer cell $\mathrm{PI} 3 \mathrm{~K} / \mathrm{Akt}$ to prove that activation of the PI3K/AKt signaling pathway is an important mechanism resulting in drug resistance of trastuzumab and identify an important target of trastuzumab drug resistance, which has provided a theoretical basis for individualized clinical therapy of moleculetargeted therapy for breast cancer, a new idea and experimental basis for clinical therapy, and is thus of clinical significance.

The authors declare no conflict of interest.

This study was supported by the Natural Science Foundation of Fujian province of China (Z0516080). 


\section{References}

1. Dębska S, Kusińska R, Czernek U, et al. Prognostic value of expression of intracellular and extracellular domains of HER2 in patients with HER2-possitive breast cancer. Wspolczesna Onkol 2011; 15: 267-73.

2. Cianfrocea M, Goldstein LJ. Prognostic and predictive factors in early-stage breast cancer. Oncologist 2004; 9: 606-616.

3. Singer CF, Köstler WJ, Hudelist G. Predicting the efficacy of trastuzumab-based therapy in breast cancer: current standards and future strategies. Biochim Binphys Acta 2008; 1786: 105-13.

4. Valabrega G, Montemurro F, Aglietta M. Trastuzumab: mechanism of action, resistance and future perspectives in HER2-overexpressing breast cancer. Ann Oncol 2007; 18: 977-84.

5. Berns K, Horlings HM, Hennessy BT, et al. A functional genetic approach identifies the PI3K pathway as a major determinant of trastuzumab resistance in breast cancer. Cancer Cell 2007; 12: 395-402.

6. Park BH, Davidson NE. PI3 kinase activation and response to Trastuzumab Therapy: what's neu with herceptin resistance? Cancer Cell 2007; 12: 297-9.

7. Narayan M, Wilken JA, Harris LN, Baron AT, Kimbler KD, Maihle NJ. Trastuzumab-induced HER reprogramming in "resistant” breast carcinoma cells. Cancer Res 2009; 69: 2191-4.

8. Faratian D, Sims AH, Mullen P, Kay C, Um I, Langdon SP, Harrison DJ. Sprouty 2 is an independent prognostic factor in breast cancer and may be useful in stratifying patients for trastuzumab therapy. PLoS One 2011; 6: e23772.

9. Kataoka Y, Mukohara T, Shimada H, Saijo N, Hirai M, Minami H. Association between gain-of-function mutations in PIK3CA and resistance to HER2-targeted agents in HER2-amplified breast cancer cell lines. Ann Oncol 2010; 21: 255-62.

10. Wiczyńska B, Rolski J. Targeted therapy. Part I. Signalling by tyrosine kinase receptors. Wspolczesna Onkol 2007; 11: 331-6.

11. Krawczyk P, Mlak R, Powrózek T, Nicoś M, Kowalski DM, Wojas-Krawczyk K, Milanowski J. Mechanisms of resistance to reversible inhibitors of EGFR tyrosine kinase in non-small cell lung cancer. Wspolczesna Onkol 2012; 16: 401-6.

12. Shoman N, Klassen S, McFadden A, Bickis MG, Torlakovic E, Chibbar R. Reduced PTEN expression predicts relapse in patients with breast carcinoma treated by tamoxifen. Mod Pathol 2005; 18: 250-9.

13. Yakes FM, Chinratanalab W, Ritter CA, King W, Seelig S, Arteaga CL. Herceptin-induced inhibition of phosphatidylinositol-3 kinase and Akt Is required for antibody-mediated effects on p27, cyclin D1, and antitumor action. Cancer Res 2002; 62: 4132-41.

14. Nicholson KM, Anderson NG. The protein kinase B/Akt signalling pathway in human malignancy. Cell Signal 2002; 14: 381-95.

15. Clark AS, West K, Streicher S, Dennis PA. Constitutive and inducible Akt activity promotes resistance to chemotherapy, trastuzumab, or tamoxifen in breast cancer cells. Mol Cancer Ther 2002; 1: 707-17.

16. Crowder RJ, Ellis MJ. Treating breast cancer through novel inhibitors of the phosphatidylinositol 3'-kinase pathway. Breast Cancer Res 2005; 7: 212-4

17. Hu L, Zaloudek C, Mills GB, Gray J, Jaffe RB. In vivo and in vitro ovarian carcinoma growth inhibition by a phosphatidylinositol 3-kinase inhibitor (LY294002). Clin Cancer Res 2000; 6: 880-6.

18. Itoh N, Semba S, Ito M, Takeda H, Kawata S, Yamakawa M. Phosphorylation of Akt/PKB is required for suppression of cancer cell apoptosis and tumor progression in human colorectal carcinoma. Cancer 2002; 94: 3127-34.

\section{Address for correspondence}

\section{Dr. Xi Chen}

Department of Medical Oncology

Fuzhou General Hospital of Nanjing Military Command

350001 Fuzhou, China

e-mail: ftczhang@163.com

Submitted: 19.10 .2012

Accepted: 15.02 .2013 